\title{
Cancer without disease
}

\section{Do inhibitors of blood-vessel growth found naturally in our bodies defend most of us against progression of cancer to a lethal stage?}

\section{Judah Folkman and Raghu Kalluri}

M any of us may have tiny tumours without knowing it. In fact, autopsies of individuals who died of trauma often reveal microscopic colonies of cancer cells, also known as in situ tumours. It has been estimated that more than one-third of women aged 40 to 50, who did not have cancer-related disease in their life-time, were found at autopsy with in situ tumours in their breast. But breast cancer is diagnosed in only $1 \%$ of women in this age range. Similar observations are also reported for prostate cancer in men. Virtually all autopsied individuals aged 50 to 70 have in situ carcinomas in their thyroid gland, whereas only $0.1 \%$ of individuals in this age group are diagnosed with thyroid cancer during this period of their life. Therefore, it has long puzzled physicians and scientists why cancer develops and progresses to be lethal only in a very small percentage of people. The realization that a lot of us carry in situ tumours, but do not develop the disease, suggests that these microscopic tumours are mostly dormant and need additional signals to grow and become lethal tumours. So, what are these additional signals, and why are most of us protected from them?

The most likely answer is our body's inherent capacity to prevent the majority of these in situ tumours from recruiting their own new blood supply, thus preventing further growth owing to a lack of oxygen and nutrients. In the absence of a new supply of blood vessels by a process known as angiogenesis, an in situ tumour can remain dormant indefinitely. Paradoxically, it is proposed that angiogenesis itself is under the control of many genes in our body known to promote cancer (oncogenes) or suppress growth of tumours (tumour suppressors) the same genes that are also involved in creating cancer cells, as encountered in the harmless in situ tumours. If the genes involved are the same, then why are most of us protected from the disease of cancer? To understand this better, we may have to define cancer as having two critical phases. In the first, acquisition of mutations, possibly due to genetic instability, leads to the transformation of normal cells in our body into cancer cells. This phase is not inherently lethal, and

is a very low incidence of solid tumours in patients with Down Syndrome, who circulate elevated levels of endostatin, an endogenous angiogenesis inhibitor, due to an extra copy of chromosome 21. An increased incidence of prostate cancer in patients with a specific polymorphism in endostatin is also noted. These examples suggest that either an increase or decrease in the angiogenic defence can alter the rate of cancer progression. Therefore, genetic control of physiological levels of endogenous angiogenesis inhibitors, such as thrombospondin-1, tumstatin and endostatin, may provide a last line of defence against the conversion of in situ tumours into a malignant

generally results in a microscopic tumour where the high rate of tumour cell division is balanced by cell death. The second phase involves a switch to the angiogenic phenotype, due to constant recruitment of new blood vessels, which converts the non-lethal in situ tumours into the expanding mass of tumour cells that is potentially lethal to an individual. Therefore, it is likely that there are critical governing factors that distinguish an individual's capacity to launch angiogenesis and enter a lethal phase of cancer.

This progression depends crucially on the balance between the in situ tumour's total angiogenic output and an individual's total angiogenic defence. The angiogenic output is contributed by growth factors such as basic FGF, VEGF, IL-8 and PDGF. An individual's defence is facilitated by endogenous angiogenesis inhibitors that are either associated with specific tissues or circulating in the blood. These inhibitors include thrombospondin, tumstatin, canstatin, endostatin, angiostatin, and interferon alpha/beta. Angiogenesis within the in situ tumour is probably initiated when the angiogenic stimulators overwhelm the host angiogenic defence. It is conceivable that such disruption in the angiogenesis balance is under the control of both the genetic make-up of any individual cancer cell and its microenvironment within the tumour. This would then explain why cancer in different individuals progresses at different rates, and also why some individuals enter the lethal phase of cancer and others do not, in spite of carrying cancer cells within their organs.

Such thinking is further fuelled by interesting occurences in nature. For instance, there phenotype of cancer. Although drugs such as Avastin, which inhibits VEGF (the growth factor involved in blood vessel development), decrease the overall angiogenic output of the tumour and result in delay of disease progression, a viable additional strategy would be to boost the angiogenic defence of an individual by providing the natural angiogenesis inhibitors as part of anti-cancer therapy.

Molecular studies aimed at a better understanding of why many of us are protected from the disease of cancer may thus lead to identification of new, non-toxic drugs capable of converting cancer into a chronic manageable disease. If - as autopsies of trauma victims reveal - a majority of us have in situ tumours, then an important goal of future research will be to prevent disease in those individuals in whom the genetics favour progression of harmless in situ tumours into a lethal form of cancer. It is possible that one day, cancer might be treated as a chronic manageable disease, such as diabetes and heart disease. Judah Folkman is at the Department of Surgery, Children's Hospital and Harvard Medical School, Boston, Massachusetts 02115, USA. Raghu Kalluri is at the Center for Matrix Biology, Department of Medicine, Beth Israel Deaconess Medical Center and Harvard Medical School, Boston,

Massachusetts 02215, USA.

\section{FURTHER READING:}

Black, W. C. \& Welch, H. G. N. Engl. J. Med. 328, 1237-1243 (1993).

Hanahan, D. \& Folkman, J. Cell 86, 353-364 (1996). Hamano, Y. et al. Cancer Cell 3, 589-601 (2003). Kalluri, R. Nature Rev. Cancer 3, 422-433 (2003). Udagawa,T. et al. FASEB J. 16,1361-1370 (2002). 